\title{
Tecnura
}

\section{Estado del agua del río Cesar por vertimientos residuales de la ciudad de Valledupar. Bioindicación por índice BMWP/Col}

\author{
State of the Cesar river water due to the residual discharge from \\ the city of Valledupar. Bioindication by BMWP/Col index.
}

\author{
Hernando Carlos Oñate Barraza', Gridis Yulieht Cortéz Henao²
}

Fecha de recepción: 2 de Diciembre de 2019

Fecha de aceptación: 6 de Mayo de 2020

Cómo citar: Oñate-Barraza., H.C., y Cortéz-Henao., G.Y. (2020). Estado del agua del río Cesar por vertimientos residuales de la ciudad de Valledupar. Bioindicación por índice BMWP/Col. Tecnura, 24(65) 39-48. DOI: $10.14483 / 22487638.15766$

\section{Resumen}

Objetivo: El objeto de este artículo es cualificar las condiciones de la corriente del río Cesar en la zona de influencia del vertimiento del STAR del municipio de Valledupar, y su relación con los indicadores de contaminación biológica e índice de calidad BMWP/Col.

Metodología: Se establecieron cuatro estaciones de muestreo sobre la corriente del río Cesar: E1 y E2, localizadas aguas arriba de la zona de mezcla de la descarga residual de la ciudad; E3, ubicada en el punto de mezcla del vertimiento y el río; y E4, situada aguas abajo de la descarga. Permitiendo conocer sus características fisicoquímicas y determinar la condición biológica de la corriente según el índice BMWP/Col para los cuatro puntos de muestreo establecidos.

Resultados: En las estaciones E1 y E2, se obtuvieron macroinvertebrados indicadores de aguas limpias y bien oxigenadas, con una clasificación de agua aceptable; mientras que las estaciones E3 y E4, indicadores de aguas contaminadas de calidad dudosa; siendo E4 la más crítica con concentraciones de oxígeno y $\mathrm{DBO}_{5}$ de 4,61 mg/l y 51,42 mg/l, respectivamente.

Conclusiones: La estación E4 evidencia la calidad de agua más contaminada según el índice aplicado, a pesar de la presencia de individuos de los géneros Hemíptera y Trichóptera que reciben una alta puntuación en el índice BMWP/Col. Se da también la aparición de grupos de macroinvertebrados indicadores de aguas de pésima calidad como los dípteros de la familia Ephydriidae, Culicidae y Chironomidae. Palabras clave: aguas residuales, bioindicadores, calidad de aguas, ecosistema lotico.

\begin{abstract}
Objective: The purpose of this article is to qualify the conditions of the Cesar river stream in the area of influence of the dumping of the STAR of the municipality of Valledupar, and its relationship with the biological indicators of contamination and BMWP/ Col quality index.
\end{abstract}

1 Magíster en Ingeniería Ambiental, ingeniero sanitario y ambiental. Docente Universidad Popular del Cesar. Valledupar, Colombia. Contacto: hernandoonate@unicesar.edu.co

2 Magíster en Ciencias Ambientales, microbióloga. Docente Colegio Bilingüe Fisher School. Valledupar, Colombia. Contacto: yugridis@ hotmail.com 
Methodology: Four sampling stations were established on the Cesar River: E1 and E2 located upstream of the mixture zone at the municipal wastewater discharge point, E3 located at the point of mixing of the discharge and the river; and E4 located downstream of the discharge, letting know its physicochemical characteristics and to determine the biological conditions of the stream according to the BMWP/Col index for each of the established stations.

Results: In stations E1 and E2, macroinvertebrates indicators of clean and well-oxygenated waters were obtained, with an acceptable water classification; while the stations E3 and E4, were found macroinvertebrates indicators of contaminated waters of doubtful quality; E4 has being the most critical area, with oxygen and $\mathrm{BOD}_{5}$ concentrations of $4.61 \mathrm{mg} / \mathrm{L}$ and $51.42 \mathrm{mg} / \mathrm{L}$ respectively.

Conclusions: The station E4 shows the quality of the most contaminated water according to the index applied, since despite the presence of individuals from the Hemiptera and Trichóptera genus that receive a high score in the BMWP/Col index, also, the appearance of groups of macro invertebrates indicators of poor quality waters such as the Diptera of the family Ephydriidae, Culicidae and Chironomidae.

Keywords: wastewater, bioindicators, water quality, lotic ecosystem.

\section{INTRODUCCIÓN}

El río Cesar en su recorrido atraviesa los departamentos de La Guajira y el Cesar, para desembocar en la ciénaga de Zapatosa la cual desemboca en el río Magdalena. En su recorrido por el departamento del Cesar, este río baña con sus aguas los municipios de Agustín Codazzi, Becerril, Bosconia, Chimichagua, Chiriguaná, Curumaní, Copey, La Jagua de Ibirico, San Diego y Valledupar, entre muchos otros. Siendo sus aguas utilizadas por estas comunidades ribereñas como fuente de abastecimiento y consumo en actividades domésticas y económicas como la agricultura, la pesca y la ganadería. Sin embargo, en el transcurso de los años se ha visto afectada la calidad de sus aguas por los vertimientos residuales de estas comunidades. El municipio de Valledupar y más específicamente el vertimiento de sus aguas residuales, ejerce un mayor impacto sobre las aguas de este afluente hídrico. Según Rodríguez, García y Pardo (2015), entre los impactos generados por las Plantas de tratamiento de Aguas Residuales (PTAR) municipales a su entorno, se pueden considerar la generación de olores, la presencia de espumas, el deterioro estético, entre otros. De igual manera, el aporte de nutrientes promueve la eutrofización que, al presentarse en exceso, induce un crecimiento desmesurado de la vegetación acuática (Peña, Muñoz y Espinosa, 2001).

En estas tres últimas décadas los efectos negativos generados sobre los recursos hídricos han llevado a una constante preocupación ambiental, que cada vez gana más adeptos. Esto ha impulsado la necesidad de mantener los ecosistemas acuáticos como un objetivo legítimo que debería ser considerado entre las distintas demandas por agua dulce (Castro, Carvajal y Céspedes, 2011).

Es tan grande el impacto negativo generado por los múltiples vertimientos (puntuales o difusos) que se ejercen sobre la corriente del río Cesar, que ha Ilevado a instituciones ambientales como Corpocesar, a declarar la emergencia ambiental en su cuenca media y baja (Resolución 0170 de 2015).

En ese sentido, el objeto de este artículo es valorar las condiciones del agua del río Cesar en la zona de influencia del vertimiento del STAR del municipio de Valledupar, y su relación con los indicadores de contaminación biológica e índice de calidad BMWP/Col.

Según Giraldo (2004), en los últimos años, la implementación del índice BMWP/Col se ha convertido en una herramienta rápida, eficaz y económica de cualificación ecológica de cuerpos de agua, en comparación con métodos tradicionales 
que se han caracterizado por su elevado costo y menor practicidad.

El método del índice BMWP/Col se estableció en Inglaterra para la identificación a nivel de familia de los grupos de macroinvertebrados haciendo uso de datos cualitativos (presencia o ausencia), puntuando en escala de 1 a 10 el nivel de tolerancia o sensibilidad a la contaminación del agua, respectivamente. La sumatoria de la puntuación obtenida, según el número de individuos de cada familia en un lugar determinado, genera el total del índice BMWP/Col.

La presencia o ausencia de macroinvertebrados bentónicos acuáticos como indicadores de contaminación es una muestra de las alteraciones fisicoquímicas de los cuerpos de agua por la acción antropogénica, pues ellos constituyen un soporte a los test de campo usualmente aplicados, lo cual representa el análisis determinante de las condiciones de una fuente hídrica (Oscoz, Campos y Escala, 2006).

Es de conocimiento que desde hace varios años se implementan diversas técnicas biológicas para evaluar la calidad del agua que son cada vez más utilizadas, sobre todo en Estados Unidos y Europa. Estas no pueden implementarse en los países tropicales sin conocer el tipo de organismos que allí habitan y sus exigencias ecológicas y distribución geográfica. Por tal razón, desde la década de 1980, Roldan (1996), el uso de un índice BMWP/ Col, como se detalla en la (tabla 1), que tiene en cuenta las familias encontradas en los ecosistemas acuáticos del departamento de Antioquia.

\section{METODOLOGÍA}

\section{Delimitación del área de estudio}

Este estudio tuvo lugar en la zona del río Cesar denominada "La descarga del Salguero", nombre derivado del puente homónimo. Esta zona coincide con el punto donde se vierte el efluente del STAR de la ciudad de Valledupar. Para la delimitación del área de estudio y la ubicación de las estaciones de muestreo se consideró que aguas arriba del punto del vertimiento residual se presentan aportes puntuales y escorrentías superficiales sobre el río que podrían afectar las características físicas, químicas y microbiológicas de este y verse reflejados en los resultados investigados, provocando un incremento en el aporte de sedimentos y la turbidez de los ríos (González, Carvajal y Loaiza, 2016). Para minimizar este efecto se establecieron cuatro estaciones sobre el río Cesar, las cuales se localizaron así: E1, 4,6 km aguas arriba del vertimiento del STAR del municipio de Valledupar y $100 \mathrm{~m}$ aguas arriba de la zona donde se presentan los aportes puntuales adicionales sobre el río Cesar; E2, 2,5 km aguas arriba del vertimiento del municipio de Valledupar, localizada aguas debajo de la zona donde se presentan los aportes puntuales adicionales sobre el río Cesar; E3, localizada en el punto de mezcla del vertimiento del STAR del municipio de Valledupar y el río Cesar; y la E4, $100 \mathrm{~m}$ aguas abajo de la descarga del STAR del municipio de Valledupar.

Tabla 1. Método BMWP/Col

\begin{tabular}{clll}
\hline Clase & \multicolumn{1}{c}{ Calidad } & \multicolumn{1}{c}{ BMWP/Col } & \multicolumn{1}{c}{ Significado } \\
\hline I & Buena & $>100$ & Aguas muy limpias a limpias \\
\hline II & Aceptable & $61-100$ & Aguas ligeramente contaminadas \\
\hline III & Dudosa & $36-60$ & Aguas moderadamente contaminadas \\
\hline IV & Crítica & $16-35$ & Aguas muy contaminadas \\
\hline V & Muy crítica & $<16$ & Aguas fuertemente contaminadas \\
\hline
\end{tabular}

Fuente: Roldán (1996). 


\section{Caracterización fisicoquímica}

Para la caracterización se realizó un muestreo compuesto en un periodo de 12 horas diarias, 2 días a la semana, durante 3 meses, donde se evaluaron los parámetros de DQO, contenido de sólidos, presencia de nutrientes inorgánicos $(\mathrm{N}$ y $\mathrm{P})$ y alcalinidad. Para los parámetros OD y DBO se aplicó un muestreo puntual en el centro de la corriente para cada una de las estaciones descritas anteriormente (Ideam, 2004).

\section{Determinar presencia/ausencia de microorganismos indicadores de contaminación del agua y el índice BMWP/Col}

El método utilizado permitió la obtención de muestras representativas de todos los taxones relativamente abundantes, para así determinar aproximadamente la densidad y biomasa para cada una de las estaciones descritas anteriormente, y poder estimar los índices en cada tramo. Para el muestreo fue utilizada una Red Surber, o red de mano, de 250-500 $\mu \mathrm{m}$ de malla, la cual se ubicó contracorriente ocupando el mayor espacio posible de la sección transversal del río, removiendo el fondo con mucho cuidado y permitiendo que los animales y sedimentos terminen dentro de la red, para depositarlos en una serie de tamices donde posteriormente fueron lavados para ser clasificados individualmente en frascos de plástico con formaldehído al 4 \%. Las muestras se llevaron al laboratorio de la Universidad Popular del Cesar donde fueron separadas en unidades sistemáticas lo más pronto posible, y se clasificaron con la ayuda de claves taxonómicas que permitieron establecer sus características ecológicas y las afectaciones morfológicas que podrían sufrir por el impacto de la contaminación en el cuerpo de agua. A continuación los individuos fueron enumerados y preservados para la determinación del índice de biomasa y de diversidad y frecuencia, para así clasificar las aguas de acuerdo con el índice BMWP/Col (Roldán, 2003)

Las puntuaciones asignadas a cada familia de macroinvertebrados según el BMWP/Col se resumen en la tabla 2.

Tabla 2. Puntuación de las familias de macroinvertebrados para obtener BMWP/Col

Familia

Siphlonuridae, Heptageniidae, Leptophebiidae Potamanthidae, Ephemeridae, Taeniopterygidae, Leuctridae, Capniidae, Perlodidae, Perlidae, Chloroperlidae, Aphelocheiridae, Phryganeidae, Molannidae, Beraeidae, Odontoceridae, Leptoceridae, Goeridae, Lepidostomatidae, Brachycentridae, Sericostomatidae, Athericidae, Blephariceridae

Astacidae, Lestidae, Calopterygidae, Gomphidae, Cordulegasteridae, Aeshnidae, Corduliidae, Libellulidae, Psychomyiidae, Philopotamidae, Glossosomatidae

Ephemerellidae, Nemouridae, Rhyacophilidae, Polycentropodidae, Limnephilidae

Neritidae, Viviparidae, Ancylidae, Hydroptilidae, Unionidae, Corophiidae, Gammaridae, Platycnemididae, Coenagriidae

Oligoneuriidae, Dryopidae, Elmidae, Helophoridae, Hydrochidae, Hydraenidae, Clambidae, Hydropsychidae, Tipulidae, Simuliidae, Planariidae, Dendrocoelidae, Dugesiidae

Baetidae, Caenidae, Haliplidae, Curculionidae, Chrysomelidae, Tabanidae, Stratiomydae, Empididae, Dolichopodidae, Dixidae, Ceratopogonidae, Anthomyidae, Limoniidae, Psychodidae, Sialidae, Piscicolidae, Hidracarina

Mesoveliidae, Hydrometridae, Gerridae, Nepidae, Naucoridae, Pleidae, Notonectidae, Corixidae, Helodidae,

Hydrophilidae, Hygrobiidae, Dysticidae, Gyrinidae, Valvatidae,, Hydrobiidae, Lymnaeidae, Physidae, Planorbidae, Bithyniidae, Sphaeridae, Glossiphoniidae, Hirudidae, Erpobdellidae, Asellidae, Ostracoda

Chironomidae, Culicidae, Muscidae, Thaumaleidae, Ephydridae

Oligochaeta (todas las clases), Syrphidae

Fuente: Roldán (2003).
Puntuación

7




\section{RESULTADOS}

\section{Características de los parámetros fisicoquí- micos en el río Cesar y el vertimiento del STAR del municipio de Valledupar}

En las tablas 3 y 4 se presentan los valores de los diferentes parámetros de calidad de agua medidos in situ y determinados analíticamente en el laboratorio de la Universidad Popular del Cesar, para las cuatro estaciones de estudio sobre el río Cesar.

Según la tabla 3, las concentraciones de saturación de oxígeno presentan su valor más bajo en la E4, donde se evidenciaron valores promedios de oxígeno disuelto de $66,07 \%$. El efecto del vertimiento genera un consumo de oxígeno alto, disminuyendo así la cantidad de oxígeno disuelto disponible en este tramo del río. Para las E1 y E2 las concentraciones de oxígeno disuelto son muy próximas a la concentración de saturación, lo cual significa que a esta distancia la carga orgánica aportada por la zona de aportes puntuales ya se ha consumido en su gran mayoría, con lo cual podemos decir que en la sección entre las E1 y E2 el río presenta una alta capacidad de autodepuración, haciendo esto que las aguas de estos sectores tengan mejores condiciones; esta zona es conocida como zona de recuperación, donde a lo largo del recorrido del río se presenta un aumento significativo del nivel de oxígeno disuelto debido a sus procesos de oxigenación. Se realizó una caracterización fisicoquímica, dentro de la cual se determinaron parámetros como: $\mathrm{DBO}_{5}, \mathrm{DQO}$, nitrógeno total, nitratos, nitritos, fósforo total, solidos suspendidos totales y alcalinidad, para cada uno de los días de muestreo y las cuatro estaciones descritas.

Según la información anterior, los aportes realizados por el STAR del municipio de Valledupar afectan las condiciones de equilibrio natural del río Cesar, alterando así el estado sanitario del mismo. El efluente del STAR, a pesar de recibir un tratamiento previo, presenta una alta concentración de materia orgánica, dependiendo del origen del vertido; para el caso de los vertimientos en estudio y sus efectos en el cuerpo receptor (río Cesar), la concentración del OD no es tan baja debido a la reaireación a la que estos efluentes son sometidos antes de mezclarse con el cuerpo receptor.

Tabla 3. Caracterización de parámetros medidos in situ

\begin{tabular}{cccccc}
\hline Estación & OD $(\mathbf{m g} / \mathbf{L})$ & $\begin{array}{c}\text { \% *saturación } \\
\text { de oxígeno }\end{array}$ & Temp. $\left({ }^{\circ} \mathbf{C}\right)$ & $\mathbf{p H}$ & Caudal $\left(\mathbf{m}^{\mathbf{3}} / \mathbf{s}\right)$ \\
\hline E1 & 6,17 & 88,45 & 28,24 & 6,75 & 4,53 \\
\hline E2 & 6,97 & 100,00 & 30,45 & 7,40 & 8,56 \\
\hline E3 & 5,98 & 85,85 & 30,19 & 7,16 & 0,31 \\
\hline
\end{tabular}

* Concentración de oxígeno de saturación 6,97 mg/L O .

Fuente: elaboración propia.

Tabla 4. Caracterización fisicoquímica del río Cesar en las estaciones de muestreo

\begin{tabular}{ccccccc}
\hline Estación & $\begin{array}{c}\text { DQO } \\
(\mathbf{m g} / \mathbf{L})\end{array}$ & $\begin{array}{c}\text { DBO } \\
(\mathbf{m g} / \mathbf{L})\end{array}$ & $\begin{array}{c}\mathbf{m g} / \mathbf{L} \\
\mathbf{N}-\mathbf{N O}_{\mathbf{3}}\end{array}$ & $\begin{array}{c}\mathbf{m g} / \mathbf{L} \\
\mathbf{N}-\mathbf{N O}_{\mathbf{2}}\end{array}$ & $\begin{array}{c}\mathbf{m g} / \mathbf{L} \\
\text { N-Total }\end{array}$ & $\begin{array}{c}\mathbf{m g} / \mathbf{L} \\
\mathbf{P}-\text { Total }\end{array}$ \\
\hline $\mathrm{E} 1$ & 62,53 & 14,00 & 1,74 & 0,39 & 2,50 & 0,37 \\
\hline $\mathrm{E} 2$ & 55,14 & 21,14 & 1,77 & 0,26 & 2,36 & 0,41 \\
\hline $\mathrm{E} 3$ & 385,21 & 150,43 & 3,10 & 0,03 & 27,05 & 4,44 \\
\hline $\mathrm{E} 4$ & 75,20 & 51,42 & 2,15 & 0,18 & 8,10 & 1,04 \\
\hline
\end{tabular}

Fuente: elaboración propia. 


\section{Aplicación del índice de calidad BMWP/Col}

A continuación se presenta la descripción de las familias encontradas en cada una de las estaciones, así como la determinación el índice de calidad BMWP/Col para cada una de ellas.

\section{E1 4,6 km aguas arriba del vertimiento del STAR del municipio de Valledupar}

Se hallaron varias comunidades de macroinvertebrados correspondientes a 8 órdenes y 12 familias que se relacionan en la tabla 5 .

Se presenta un listado de macroinvertebrados indicadores de aguas limpias y bien oxigenadas; y de aguas limpias y frías. Los ephemeróteros y plecópteros son buenos indicadores de tal condición del agua. Ellos presentan agallas laterales que toman el oxígeno disuelto del agua en corrientes medias y rápidas. Los trichopteros de la familia $\mathrm{Hy}$ dropsychidae son buenos bioindicadores de aguas limpias, ya que viven en aguas con altas concentraciones de oxígeno disuelto (aproximadamente 89 \% de saturación de oxígeno).

\section{E2 2,5 km aguas arriba del vertimiento del municipio de Valledupar}

Se hallaron varias comunidades de macroinvertebrados correspondientes a 7 órdenes y 12 familias que se relacionan en la tabla 6.

La mayoría de macroinvertebrados acuáticos encontrados en la estación de muestreo E2 son indicadores de aguas limpias, bien oxigenadas; esto se confirma con el porcentaje de saturación de oxígeno disuelto en la E2 (ver tabla 3), que evidencia fenómenos muy leves de contaminación. Algunos ephemeropteros y trichopteros son tolerantes a elevadas concentraciones de sólidos suspendidos, y se hallan en aguas limpias a medianamente turbias.

\section{E3 localizada en el punto de mezcla del vertimiento del STAR del municipio de Valledupar y el río Cesar}

La evaluación para los vertimientos se realizó en la zona de mezcla, encontrando 3 órdenes y 10 familias. Los resultados que se describen en la tabla 7 , y son ratificados por la evaluación de parámetros fisicoquímicos descritos anteriormente.

Tabla 5. Macroinvertebrados E1

\begin{tabular}{ccc}
\hline Orden & Familia & Puntaje (índice BMWP/Col) \\
\hline Trichoptera & Hidropsychidae & 7 \\
Hemiptera & Naucoridae & 8 \\
\cline { 2 - 3 } Ephemeroptera & Vellidae & 7 \\
\cline { 2 - 3 } & Baetidae & 7 \\
\cline { 2 - 3 } & Leptohyphidae & 9 \\
\hline \multirow{nyy}{*}{ Odonata } & Leptophlebidae & 8 \\
\hline \multirow{nyy}{*}{ Coleoptera } & Coenagríonidae & 6 \\
\hline Díptera & Libellulidae & 7 \\
\hline Plecoptera & Elmidae & 2 \\
\hline Lepidoptera & Dryopidae & 10 \\
\hline
\end{tabular}

Fuente: elaboración propia. 
Tabla 6. Macroinvertebrados E2

\begin{tabular}{|c|c|c|}
\hline Orden & Familia & Puntaje (índice BMWP/Col) \\
\hline Trichoptera & Hidropsychidae & 6 \\
\hline \multirow{2}{*}{ Decapoda } & Trichodactylidae & 6 \\
\hline & Palaemonidae & 6 \\
\hline \multirow{2}{*}{ Hemiptera } & Naucoridae & 8 \\
\hline & Vellidae & 7 \\
\hline \multirow{3}{*}{ Ephemeroptera } & Baetidae & 6 \\
\hline & Leptohyphidae & 7 \\
\hline & Leptophlebidae & 9 \\
\hline \multirow{2}{*}{ Odonata } & Coenagríonidae & 8 \\
\hline & Libellulidae & 6 \\
\hline Coleoptera & Elmidae & 7 \\
\hline Diptera & Culicidae & 2 \\
\hline \multicolumn{2}{|c|}{ Total } & 78 \\
\hline
\end{tabular}

Fuente: elaboración propia.

Tabla 7. Macroinvertebrados E3

\begin{tabular}{|c|c|c|}
\hline Orden & Famila & Puntaje (índice BMWP/Col) \\
\hline \multirow{5}{*}{ Diptera } & Culicidae & 2 \\
\hline & Psychodidae & 2 \\
\hline & Ephydridae & 2 \\
\hline & Chironomidae & 2 \\
\hline & Stratiomyidae & 4 \\
\hline \multirow{2}{*}{ Hemiptera } & Corixidae & 4 \\
\hline & Belostomatidae & 4 \\
\hline \multirow{3}{*}{ Coleoptera } & Dytiscidae & 8 \\
\hline & Noteridae & 6 \\
\hline & Hidrophilidae & 3 \\
\hline \multicolumn{2}{|c|}{ Total } & 37 \\
\hline
\end{tabular}

Fuente: elaboración propia.

A través del cálculo del índice BMWP/Col, se obtuvieron valores de calidad de agua de 37, lo cual indica que según la tabla 1, son aguas contaminadas de calidad dudosa y que pertenecen al tipo IV en la zona de mezcla entre el vertimiento del sistema de tratamiento de aguas residual del municipio de Valledupar y el río Cesar.
E4 $100 \mathrm{~m}$ aguas abajo de la descarga del STAR del municipio de Valledupar

Se hallaron varias comunidades de macroinvertebrados correspondientes a 6 órdenes y 12 familias que se relacionan en la tabla 8 . 
Tabla 8. Macroinvertebrados E4

\begin{tabular}{|c|c|c|}
\hline Orden & Familia & Puntaje (índice BMWP/Col) \\
\hline \multirow{3}{*}{ Díptera } & Chironomidae & 2 \\
\hline & Ephydridae & 2 \\
\hline & Culicidae & 2 \\
\hline \multirow{2}{*}{ Hemiptera } & Vellidae & 7 \\
\hline & Gerridae & 7 \\
\hline Trichoptera & Leptoceridae & 8 \\
\hline \multirow{2}{*}{ Coleoptera } & Noteridae & 4 \\
\hline & Lymnaeidae & 4 \\
\hline Haplotaxida & Tubificidae & 1 \\
\hline Total & & 37 \\
\hline
\end{tabular}

Fuente: elaboración propia.

A pesar de la presencia de individuos de los géneros Hemíptera y Trichoptera que reciben una alta puntuación en el índice BMWP/Col, también se evidencia la aparición de grupos de macroinvertebrados indicadores de aguas de pésima calidad, como los dípteros de la familia Ephydriidae, Culicidae y Chironomidae. Es importante aclarar que, para la E4, el nivel de oxígeno disuelto se sitúa en 4,61; lo que corresponde a aguas en condiciones críticas, sin embargo, el índice de calidad BMWP/Col arroja como resultado un agua clase IV (dudosa), estas diferencias son justificadas con la presencia de individuos de la familia Leptoceridae, que si bien están presentes en aguas oxigenadas también pueden desarrollarse en condiciones de abundante disponibilidad de materia orgánica, como se observa en la caracterización fisicoquímica (tabla 4). Una vez obtenidos los valores del índice BMWP/Col para la evaluación de calidad de agua en cada uno de los tramos de estudio, se muestran los resultados generales y su relación con las variables oxígeno disuelto $\left(\mathrm{O}_{2}\right)$ y $\mathrm{DBO}_{5}$ con los cuales se ratifican los resultados del índice BMWP/ Col, para todos los casos (tabla 9).

Tabla 9. Resultados generales

\begin{tabular}{|c|c|c|c|c|c|c|c|}
\hline Estación & km & $\underset{(\mathbf{m g} / \mathrm{L})}{\mathrm{O}_{3}}$ & $\begin{array}{l}\mathrm{DBO}_{5} \\
(\mathrm{mg} / \mathrm{L})\end{array}$ & Clase & Significado & Calidad & Color \\
\hline E1 & 0 & 6,17 & 14 & III & $\begin{array}{l}\text { Aguas medianamente } \\
\text { contaminadas }\end{array}$ & Aceptable & \\
\hline E2 & 2,528 & 6,97 & 21,14 & III & $\begin{array}{l}\text { Aguas medianamente } \\
\text { contaminadas }\end{array}$ & Aceptable & \\
\hline E3 & 4,626 & 5,98 & 25,93 & IV & Aguas contaminadas & Dudosa & \\
\hline E4 & 4,726 & 4,61 & 51,42 & IV & Aguas contaminadas & Dudosa & \\
\hline
\end{tabular}

Fuente: elaboración propia. 


\section{CONCLUSIONES}

Los flujos másicos resultados de la caracterización de las estaciones $\mathrm{E} 1=5480,85 \mathrm{~kg} / \mathrm{d}$, y $\mathrm{E} 2=14164,57 \mathrm{~kg} / \mathrm{d}$, indican una mezcla completa $\mathrm{y}$ el aporte de carga que se ejerce entre el río y las descargas puntuales que se encuentra entre estas estaciones; esto se evidencia en la estación E2, donde el flujo másico presenta un incremento debido al aporte por escorrentías superficiales. Sin embargo, para los puntos E2 y E4, los valores fueron $14164,57 \mathrm{~kg} / \mathrm{d}$ y $28320,45 \mathrm{~kg} / \mathrm{d}$, respectivamente, indicando que la mezcla de los vertimientos del STAR del municipio de Valledupar no es completa a la distancia de 100 m en los cuales se realizó la evaluación.

La E4 presenta la calidad de agua más contaminada en el tramo, según el índice BMWP/Col, ya que a pesar de la presencia de individuos de los géneros Hemíptera y Trichóptera que reciben una alta puntuación en el índice BMWP/Col, también aparecen grupos de macroinvertebrados indicadores de aguas de pésima calidad, como los dípteros de la familia Ephydriidae, Culicidae y Chironomidae.

En la E4 el nivel de oxígeno disuelto se sitúa en 4,61 , lo que corresponde a aguas en condiciones críticas; sin embargo, el índice de calidad BMWP/ Col arroja como resultado un agua clase IV (dudosa). Estas diferencias son justificadas con la presencia de individuos de la familia Leptoceridae, que si bien están presentes en aguas oxigenadas también pueden desarrollarse en condiciones de abundante disponibilidad de materia orgánica.

El método BMWP/Col representa una herramienta de gran utilidad para la bioindicación de la calidad de cuerpos de agua de media y baja densidad especialmente adaptado a los ecosistemas de la región Andina colombiana y aplicado a diversas regiones del país teniendo en cuenta las características del cuerpo de agua. Por tanto, es importante avanzar y profundizar en el estudio ecológico y taxonómico de macroinvertebrados bénticos como indicadores de calidad del agua especialmente en la región de la costa Atlántica de Colombia, donde la información disponible sobre estos organismos para el uso mencionado es limitada.

La correlación de los resultados obtenidos a partir del método BMWP/Col para el río Cesar, en los tramos de estudio con las características fisicoquímicas, microbiológicas y comportamiento de constantes cinéticas de la zona, constituye un antecedente de gran relevancia para posteriores investigaciones en la región.

\section{FINANCIAMIENTO}

Esta investigación se desarrolló en el marco de convocatoria interna para la financiación de proyectos de grupos de investigación de la Universidad Popular del Cesar.

\section{REFERENCIAS}

Castro Heredia, L., Carvajal Escobar, Y. y Céspedes López, G. (2011). Aplicación de la metodología de caudales básicos de mantenimiento en la cuenca media del río Tuluá-subcuenca del río Cauca. Tecnura, 16(31), 33-44. DOI:10.14483/udistrital.jour. tecnura.2012.1.a03

Giraldo Quintero, G. (2004). Bioindicación de la calidad del agua en corrientes alto andinas. Aplicación de los índices de monitoreo biológico $\mathrm{BMWP}^{\prime} /$ Col y BMWP'/Cu. Tecnura, 7(14), 17-23. DOI: 10.14483/22487638.6172

González López, N., Carvajal Escobar, Y. y Loaiza Cerón, W. (2016). Análisis de sequías meteorológicas para la cuenca del río Dagua, Valle del Cauca, Colombia. Tecnura, 20(48), 101-113. DOI: 10.14483/ udistrital.jour.tecnura.2016.2.a07

Instituto de Hidrología, Meteorología y Estudios Ambientales (Ideam) (2004). Guía para monitoreo y seguimiento del agua. Bogotá.

Oscoz, J., Campos, F. y Escala, M. (2006). Variación de la comunidad de macroinvertebrados bentónicos en relación con la calidad de las aguas. Limnetica, 25(3), 683-692. 
Peña Prieto, L., Muñoz Martínez, M. y Espinosa Correa, A. (2001). Tratamiento de aguas residuales municipales y su impacto ambiental sobre un ecosistema, Universidad Distrital, Colombia. Tecnura, 5(9), 3545. DOI: 10.14483/22487638.6116

Resolución 0170 del 09 de marzo de 2015. Por medio del cual se declara la emergencia ambiental en la cuenca media y baja del río Cesar, se adoptan medidas de emergencia ambiental y se establecen otras disposiciones, Corpocesar.
Rodríguez Miranda, J. P., García Ubaque, C. A. y Pardo Pinzón, J. (2015). Selección de tecnologías para el tratamiento de aguas residuales municipales. Tecnura, 19(46), 149-164. DOI: 10.14483/udistrital. jour.tecnura. 2015.4.a12

Roldán, G. (2003). Bioindicación de la calidad del agua en Colombia. 1a. ed. Medellín: Editorial Universidad de Antioquia.

Roldan, G. (1996). Guía para el estudio de macroinvertebrados acuáticos del departamento de Antioquia. Medellín: Colciencias, Universidad de Antioquia. 